\title{
Transplantation of placenta-derived multipotent cells in rats with dimethylhydrazine-induced colon cancer decreases survival rate
}

\author{
HANNA SVITINA $^{1,2}$, INESSA SKRYPKINA ${ }^{1,3}$, PAVLO ARESHKOV ${ }^{1,3}$, VITALIY KYRYK ${ }^{4}$, TETIANA BUKREIEVA ${ }^{1}$, \\ PAVLO KLYMENKO $^{4}$, LIUDMYLA GARMANCHUK ${ }^{2}$, GALYNA LOBINTSEVA $^{1}$ and VOLODYMYR SHABLII ${ }^{1,2}$ \\ ${ }^{1}$ Cell Culture Laboratory, Cryobank, Institute of Cell Therapy, Kyiv 03126; ${ }^{2}$ Department of Biochemistry, \\ Educational and Scientific Centre 'Institute of Biology and Medicine', Taras Shevchenko National University of Kyiv, \\ Kyiv 01601; ${ }^{3}$ Department of Functional Genomics, Institute of Molecular Biology and Genetics of \\ National Academy of Science of Ukraine, Kyiv 03680; ${ }^{4}$ Department of Cell and Tissue Technologies, \\ State Institute of Genetic and Regenerative Medicine of National Academy of Medicine of Ukraine, Kyiv 04114, Ukraine
}

Received September 8, 2017; Accepted January 22, 2018

DOI: $10.3892 / \mathrm{ol} .2018 .7996$

\begin{abstract}
Transplantation of placenta-derived multipotent cells (PDMCs) is a promising treatment method for many diseases. However, the impact of PDMCs on colon cancer has not yet been studied. PDMCs were obtained from rat placentas by culturing tissue explants. Colon cancer was experimentally induced in male albino Wistar rats by administering $20 \mathrm{mg} / \mathrm{kg}$ dimethylhydrazine (DMH) once a week for 20 consecutive weeks. The administration of the PDMCs was performed at the 20th week after the first DMH injection. The number and size of each tumour lesion were calculated in the 5th week after transplantation. The tumour type was determined by standard histological methods. To study the engraftment of PDMCs in the body of rats, the cells were transduced with enhanced green fluorescent protein. Cell engraftment was determined by assessing the presence of EGFP by PCR and immunohistochemistry. Survival of all rats was monitored daily. Allogeneic transplantation of PDMCs to rats at middle phase of DMH-induced colon carcinogenesis did not significantly influence the number of neoplasms and the parameters of mean and total tumour area, but led to an increase in size of the most invasiveness tumours. Intravenous allogeneic
\end{abstract}

Correspondence to: Dr Hanna Svitina or Dr Volodymyr Shablii, Cell Culture Laboratory, Cryobank, Institute of Cell Therapy, 3 Kosmonavta Komarova Avenue, Kyiv 03126, Ukraine

E-mail: anja.onishchenko@gmail.com

E-mail: v_shabliy@ukr.net; v.shabliy@stemcellclinic.com.ua

Abbreviations: MSCs, mesenchymal stem cells; PDMCs, placenta-derived multipotent cells; CC, colorectal cancer; EGFP, enhanced green fluorescent protein; PCR, polymerase chain reaction; SCID, severe combined immunodeficiency; DMH, dimethylhydrazine; DMEM, Dulbecco's modified Eagle's medium; FBS, fetal bovine serum; rPDMCs, rat PDMCs

Key words: PDMCs, MSCs, colon cancer, dimethylhydrazine transplantation of PDMCs reduced the survival rate of rats with colon cancer by 17 days. PDMCs from rats engrafted into tissues of the normal intestine, tumours, lungs, liver, and spleen of rats for five weeks after intravenous transplantation. These results suggest that intravenous allogeneic transplantation of PDMCs promotes colon cancer progression and has a negative impact on survival of rats.

\section{Introduction}

Transplantation of mesenchymal stem cells (MSCs) is a promising treatment for many diseases (1). However, the effect of transplantation of MSCs on the development of cancer remains controversial. The influence of MSCs on tumour development was studied using in vitro co-cultivation (2) and xenotransplantation models, where human-derived stem cells were transplanted into study animals (3). Controversial effects of MSCs on cancer might be related to differences in approaches towards cancer modelling, e.g., chemical induction (4) or xenotransplantation of cancer cell lines (5).

Colorectal cancer (CC) is the second major type of cancer in Europe and the third major type in the USA (6). Symptoms of the disease appear only at later stages of the tumour, hence only $39 \%$ of the cases are diagnosed at early stages, and prognosis of the disease development remains unfavourable for the remaining cases, with the five-year survival rate of 11-69\% (7).

Dimethylhydrazine (DMH)-induced CC rat model follows the initiation and development processes of human spontaneous colon tumours closely compared to those followed by the xenotransplantation models of human CC cells and mouse cell lines exhibiting spontaneous carcinogenesis (8). The DMH model follows the morphological and molecular stages of CC in humans at every stage of tumour development (9). Under the influence of DMH, mutations occur in the proto-oncogenes, Apc, Kras, and Trp53, in the colonocytes of rats. Similar mutations also cause CC in humans. Additionally, like human CC cells, DMH-induced cancer cells show microsatellite instability and chromosomal instability (9). Chemically induced $\mathrm{CC}$ allows one to study other aspects including natural invasiveness of tumours into the intestine wall, and the engraftment 
of intravenously administered MSCs in spontaneous tumours, which cannot be observed when models of xenotransplantation with $\mathrm{CC}$ cell lines are used.

Placenta-derived multipotent cells (PDMCs) satisfy the criteria of International Society for Cellular Therapy (10), but exhibit some features that distinguish them from other MSCs $(11,12)$. The placenta, as source of MSCs, has a number of advantages; no invasive procedures are required to obtain the cells, no moral and ethical issues arise, and there is no lack of cell material. The safety and efficiency of using PDMCs to treat intestinal inflammation have already been established in clinical trials (13). Despite these positive results, the effect of PDMCs on CC that often occurs together with inflammatory processes has not been sufficiently investigated.

Previous study showed that intravenous infusion of human and rat PDMCs did not affect the number and dimensions of DMH-induced colon tumours in rats (14). Intravenous administration was chosen because it is the most widely used technique of MSC transplantation in clinical practice $(15,16)$. Therefore, the aim of this study was to investigate the effects of allogeneic intravenous transplantation of PDMCs in the middle phase of colon carcinogenesis on the progression of different types of colon tumours and rat survival.

\section{Materials and methods}

Isolation and culture of rat PDMCs. All animal experiments were performed in accordance with the international guidelines of the European Convention for the Protection of Vertebrate Animals used for experimental and other scientific purposes (European Convention, Strasburg, 1986) and Article 26 of the Law of Ukraine 'On protection of animals from cruelty' (no. 3447-IV, 21.02.2006), in addition to following all norms of bioethics and biosafety. The protocol was approved by The Bioethics Committee of The Educational and Scientific Centre 'Institute of Biology and Medicine' of Taras Shevchenko National University of Kyiv (Protocol no. 8,03.04.14). Female albino rats $(n=5)$ were euthanized on the 21st day of pregnancy through carbon dioxide asphyxiation, and the placentas were immediately collected and processed. Briefly, the $\mathrm{CO}_{2}$ flow rate was started with $8 \mathrm{l} / \mathrm{min}$ in a 30 -litre chamber. As unconsciousness occurred as indicated by the loss of the righting reflex, the flow of the gas was increased to $12 \mathrm{l} / \mathrm{min}$ to fill the chamber and decrease the time to death. $\mathrm{CO}_{2}$ flow was maintained for at least $1 \mathrm{~min}$ after respiratory arrest. Death was confirmed when the animal was unresponsive to a toe pinch. Placentas from male foetuses were selected $(n=22)$.

PDMCs were isolated from the subculture of placental explants outgrowth cells as described previously (14). Placental tissue fragments were placed in cell culture dishes (Sarstedt, Nümbrecht, Germany) containing high-glucose Dulbecco's modified Eagle's medium (DMEM; Life Technologies Co., Paisley, UK) supplemented with $7 \%$ foetal bovine serum (FBS; HyClone; GE Healthcare Life Sciences, Logan, UT, USA) and 3\% allogeneic rat serum (BioWest, Nuaillé, France). Primary cultures were cultivated in standard conditions, i.e., in a humidified atmosphere at $37^{\circ} \mathrm{C}$ and $5 \% \mathrm{CO}_{2}$, and the culture medium was changed twice a week.
Generation of PDMC lines expressing green fluorescent protein $(G F P)$ using lentiviruses. Lentiviral transfer vector pCDH-CMV-MCS-EF1-copGFP (System Biosciences, Mountain View, CA, USA) and psPAX2, pMD2. G packaging plasmids were kindly provided by Dr. D. Trono (Ecole Polytechnique Federale de Lausanne, Lausanne, Switzerland) (17). EGFP sequence was inserted into transfer vector pCDH-CMV-MCS-EF1-copGFP after PCR amplification of the corresponding DNA fragment (786 bp) using pEGFP-C1 plasmid as a template and specific primers (forward, 5'-TCCGCTAGCGCTACCGGTCGCCACC-3' and reverse, 5'-GAGAATTCATCAGTTATCTAGAAGCTTGAG CTCGA-3') containing NheI and EcoRI endonuclease restriction sites respectively.

293 T cells were seeded on a $100-\mathrm{cm}$ tissue culture dish and incubated until they reached $\sim 70 \%$ confluence. $2 \mathrm{~h}$ prior to transfection cell medium was replaced with $10 \mathrm{ml}$ of fresh DMEM supplemented with $10 \%$ FBS. $5 \mu \mathrm{g}$ of EGFP-containing transfer vector same as 3,5 and 1,5 $\mu \mathrm{g}$ of packaging plasmids (psPAX2 and pMD2.G, respectively) were diluted in $1 \mathrm{ml}$ of serum-free DMEM with subsequent addition of $25 \mu \mathrm{l}$ of briefly vortexed TurboFect ${ }^{\mathrm{TM}}$ Transfection Reagent (Thermo Fisher Scientific, Inc., Vilnius, Lithuania). After pipetting and $20 \mathrm{~min}$ incubation, transfection reagent/DNA mixture was dropwise added to 293T cells. $5 \mathrm{~h}$ later cell growth media was changed and incubation was continued for 2 days. Post the 48-h incubation, cell culture supernatant was transferred to $15 \mathrm{ml}$ tube and centrifuged at 3,000 $\mathrm{x}$ g for $10 \mathrm{~min}$ and then passed through a $0.22 \mu \mathrm{M}$ ultra-low protein-binding Millex-GV Syringe Filter (Durapore $^{\circledR}$; Merck Millipore, Cork, Ireland).

The PDMCs were transduced twice with virus-containing supernatant plus polybrene at the final concentration of $8 \mu \mathrm{g} / \mathrm{ml}$ (Sigma-Aldrich; Merck KGaA, Darmstadt, Germany) overnight and media were changed between transductions.

Flow cytometry. The number of GFP-expressing PDMCs $(\mathrm{n}=4)$ was determined $24 \mathrm{~h}$ after transduction. Cells were harvested by trypsinization, resuspended in DMEM supplemented with $1 \%$ FBS, passed through $70 \mu \mathrm{m}$ cell strainer (BD Biosciences, Franklin Lakes, NJ, USA) and analysed on a BD FACSAria cell sorter using FACS Diva 6.1.2 software (both from BD Biosciences). To measure the viability of PDMCs, cells were incubated with $5 \mu \mathrm{l}$ of 7-AAD (BD Pharmingen, San Diego, CA, USA) for $10 \mathrm{~min}$ at $4^{\circ} \mathrm{C}$ in the dark. Using forward and side scatter parameters we eliminated debris from the analysis. GFP and 7-AAD were excited by argon blue laser $(488 \mathrm{~nm})$ and fluorescence was detected in the FITC channel using a 530/30 nm bandpass filter and in PerCP-Cy5.5 channel using 695/40 $\mathrm{nm}$ bandpass filter respectively. PDMCs without transduction were used as negative control. Single-stained samples (7-AAD single-stained cells without transduction and GFP-expressing cells without 7-AAD) were used to adjust the compensation of fluorochrome overlap. At least 10.000 cells were analyzed for each sample.

\section{CC modelling and PDMC administration}

In vivo experimental design. Experiments were performed using 4-month-old male albino Wistar rats $(n=137)$, weighing 180-200 g, obtained from the Central Animal House of the Institute of Pharmacology and Toxicology of NAMS of 
Ukraine. The animals were maintained under 12-h light/dark cycle, $60 \%$ humidity at $20-22^{\circ} \mathrm{C}$ and fed on standard diet and tap water ad libitum. DMH (Sigma-Aldrich; Merck KGaA) was dissolved in physiological saline (PS) and adjusted to pH 6.5 with $2 \mathrm{M}$ sodium hydroxide immediately prior to use. To induce tumour development, rats were subcutaneously injected with $20 \mathrm{mg} / \mathrm{kg}$ DMH in $0.1 \mathrm{ml}$ PS once weekly for 20 weeks $(\mathrm{n}=122)$ as described previously (9). Intact control animals $(n=15)$ received PS subcutaneously once a week for 20 weeks.

At the 20th week of modelling, rats with DMH-induced $\mathrm{CC}$ were sacrificed (group base, $\mathrm{n}=11$ ) to confirm the initiation of tumour development equivalent to stage $T_{1-2} \mathrm{~N}_{0-1} \mathrm{M}_{0}$ of CC in humans. To confirm this, a thorough histological analysis of the developing tumours was conducted.

Further, at the start of the 20th week, rats with DMH-induced cancer were intravenously administered with $0.5 \mathrm{ml}$ PS (group PS, $\mathrm{n}=13$ ), or PDMCs (group PDMCs, $\mathrm{n}=13$ ) from four rat fetuses (4 placenta tissue samples) at a dose of $2.2 \times 10^{6} / \mathrm{kg}$ body mass of rat in $0.5 \mathrm{ml}$ PS (Fig. 1). The rats were sacrificed at the 25 th week.

Evaluation of colon lesions. The tumour nodule sizes were calculated and histological analysis was performed as described by Svitina et al (14). Briefly, the rats were euthanized through carbon dioxide asphyxia, after which their abdomens were opened, and their entire gastrointestinal tracts were removed and cut longitudinally. The colon lesions were defined macroscopically and colon was photographed with the ruler at least three times. The $10 \mathrm{~mm}$ bar was set in each photo, lesions were counted, and their area was calculated using ImageJ 1.46r software (National Institutes of Health, Bethesda, MD, USA). The mean from no less than three photos was calculated for each lesion. The total tumour area per animal was calculated as the sum of areas of all lesions in the colon. The average tumour area was calculated by dividing of total tumour area on number of lesions per rat. Tissue lesions were excised for standard H\&E histological analysis (14).

To analyse the distribution of tumours by the degree of invasion, every tumour was assigned a certain value from 0 to 4 according to the international tumour classification system, TNM: T0, (Tis) carcinoma in situ, the tumour is located in the epithelial layer; T1, the tumour reaches submucosa; T2, the tumour grows to the muscular layer; T3, the tumour infiltrates tela subserosa; T4, the tumour grows into the visceral peritoneum or other organs. The grade of tumour was evaluated depending on the state of epithelium differentiation: well-differentiated tumour: the epithelium contains all types of colon epithelium (epitheliocytes and goblet cells) and reflects native colon crypt structure; moderatory: crypt get deformed, epithelium get multilayer structure; poorly: there are no native structure and gland forming.

Survival analysis. Colon cancer was induced as described above. At the start of the 20th week the rats with DMH-induced CC were intravenously administered $0.5 \mathrm{ml}$ PS (group PS-S, $\mathrm{n}=28$ ), or PDMCs (group PDMCs-S, $\mathrm{n}=47$ ) from three pregnant rats (14 placenta tissue samples) at a dose of $2.2 \times 10^{6} / \mathrm{kg}$ body mass of rat in $0.5 \mathrm{ml}$ PS. Survival of all the rats was noted daily until death. None of the rats had to be sacrificed because of excessive bleeding, open wound infection, or moribund status.

Cell engraftment assay. Spreading of PDMCs-GFP was studied using healthy rats and those with cancer. At the start of the 20th week, rats with DMH-induced CC were intravenously administered with PDMCs-GFP (Group DMH + PDMCs-GFP, $\mathrm{n}=5$ ) from two pregnant rats (3 placenta tissue samples), or $0.5 \mathrm{ml}$ PS (Group DMH + PS, n=5), and intact rats received PDMCs-GFP (Group Int + PDMCs-GFP, $n=5$ ) from two pregnant rats (3 placenta tissue samples) (Fig. 1).

The cells were administered intravenously at a dose of $2.2 \times 10^{6} / \mathrm{kg}$ body mass of rat in $0.5 \mathrm{ml}$ PS. The rats were euthanized at the 21st week through carbon dioxide asphyxiation and the tumour and colon tissue (from healthy rats) fragments, liver, lungs, spleen, and kidneys were extracted. PDMCs engraftment was studied by PCR to contain EGFP DNA. Immunohistochemistry was used to analyse localisation of engrafted cells in colon tumour tissue.

DNA isolation and polymerase chain reaction (PCR). For PCR analysis, tissue samples were frozen in liquid nitrogen and stored at $-70^{\circ} \mathrm{C}$. Genomic DNA was extracted according to the standard phenol-chloroform extraction procedure with modifications as described previously (14). Extracted DNA was stored at $-20^{\circ} \mathrm{C}$ once the concentration evaluation was performed using NanoDrop 2000 (Thermo Fisher Scientific, Inc., Wilmington, DE USA). The quality of the isolated DNA was verified by PCR for the $\beta$-actin gene (forward, 5'-ACC AACTGGGACGATATGGAGAAGA-3' and reverse, 5'-TAC GACCAGAGGCATACAGGGACAA-3'), and the PCR products were examined by electrophoresis. After amplification, two products were obtained: $680 \mathrm{bp}$ (corresponding to the $A c t b$ gene) and 214 bp (low specific of Actal gene). A 533 bp region of the $e G F P$ transgene was amplified using the following primers: Forward, 5'-CCGCTAGCGCTACCGGTCGCC ACC-3' and reverse, 5'-GGCGGATCTTGAAGTTCACC-3'.

PCR reactions were performed on an Applied Biosystems 2720 thermal cycler (Applied Biosystems; Thermo Fisher Scientific, Inc.) to final volumes of $20 \mu \mathrm{l}$ containing $1 \mathrm{X}$ Hot Start PCR buffer $\left(2.0 \mathrm{mM} \mathrm{Mg}^{2+}\right), 0.2 \mathrm{mM}$ dNTPs, $0.3 \mu \mathrm{M}$ of each primer and 1.25 units of Maxima Hot Start Taq DNA polymerase (Thermo Fisher Scientific, Inc., Vilnius, Lithuania). Each sample was assayed in triplicate, and each run included water blanks. PCR conditions were as follows: $95^{\circ} \mathrm{C}$ for $4 \mathrm{~min}$, then 40 cycles of $95^{\circ} \mathrm{C}$ for $40 \mathrm{sec}, 59^{\circ} \mathrm{C}$ for $20 \mathrm{sec}$ and $72^{\circ} \mathrm{C}$ for $40 \mathrm{sec}$, with a final extension for $7 \mathrm{~min}$ at $72^{\circ} \mathrm{C}$. PCR products were analysed using ethidium bromide stained $1 \%$ agarose gels electrophoresis. Weight Marker 'GeneRuler DNA Ladder Mix' (Thermo Fisher Scientific, Inc.) and the ladder were supplied with 6X DNA Loading Dye. Detection of the gel images was performed using a ChemiDoc ${ }^{\mathrm{TM}} \mathrm{XRS}+$ System (Bio-Rad Laboratories, Inc., Hercules, CA, USA).

Immunohistochemistry. Following PDMCs administration, colon tumours from DMH-treated rats (Group DMH + PDMCs-GFP; $n=4$ ) were fixed in $10 \%$ buffered formalin at $4^{\circ} \mathrm{C}$ for $24 \mathrm{~h}$, embedded in paraffin and sectioned on 5- $\mu \mathrm{m}$ thick slices using Microm HM325 microtome (Carl 


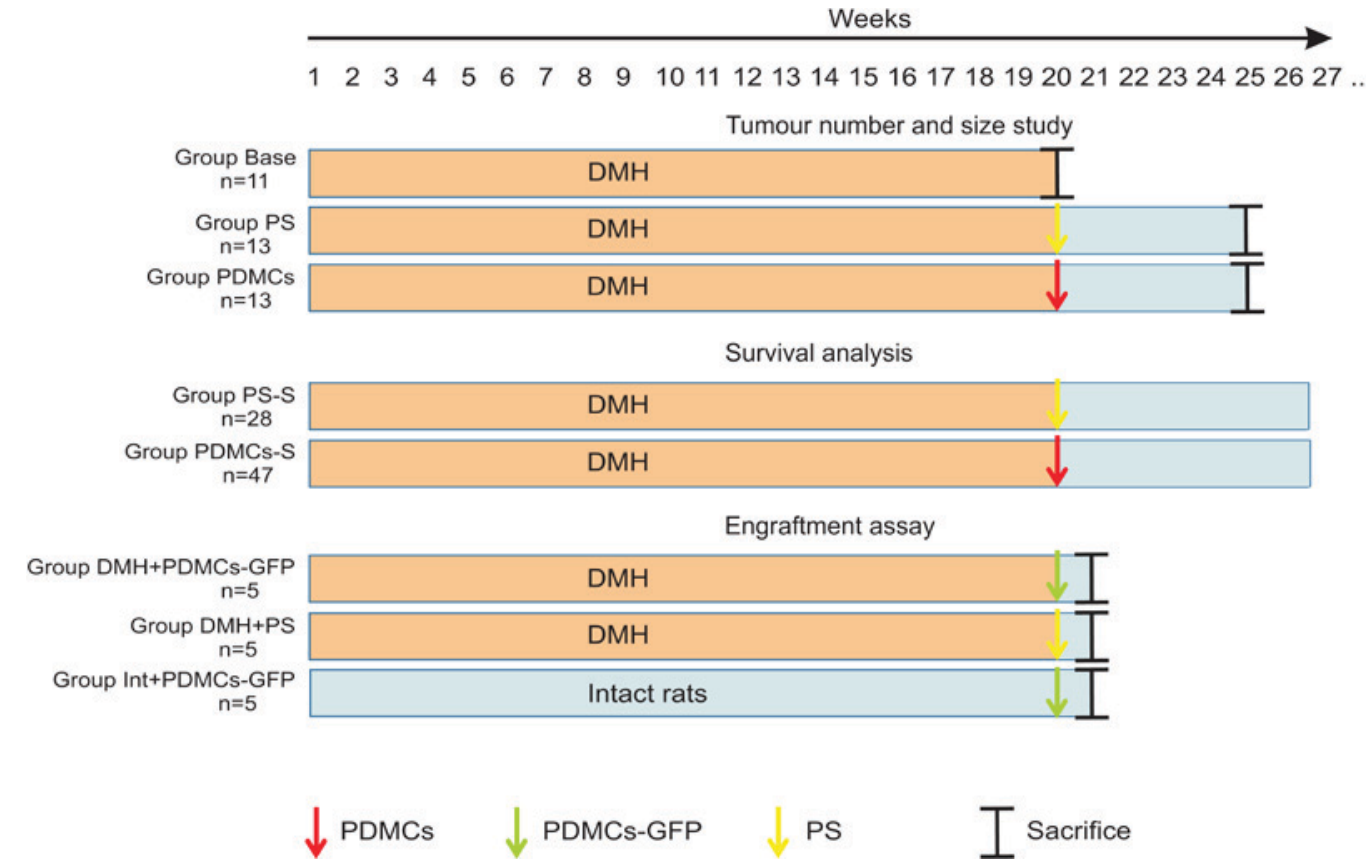

Figure 1. Treatment protocols for the different study groups. Group Base received DMH subcutaneously at a dose of $20 \mathrm{mg} / \mathrm{kg}$ once a week for 20 weeks. At the start of 21th week, the animals were sacrificed to confirm tumour formation; Group PS received DMH subcutaneously at a dose of $20 \mathrm{mg} / \mathrm{kg}$ once a week for 20 weeks. At the start of 20th week, the animals were injected with $0.5 \mathrm{ml} \mathrm{PS}$ and then maintained without supplements for 5 weeks of the experimental period. At the end of 25th week, rats were sacrificed. Group PDMCs was treated similarly to Group PS; however, at the start of week 20, PDMCs were intravenously injected at a dose of $2.2 \times 10^{6} / \mathrm{kg}$ body mass of rat in $0.5 \mathrm{ml}$ PS. Group PS-S was treated similarly to Group PS; however, all animals were used for survival analysis; Group PDMCs-S was treated similarly to Group PDMCs; however, all animals were used for survival analysis. Group DMH + PDMCs-GFP received DMH subcutaneously at a dose of $20 \mathrm{mg} / \mathrm{kg}$ once a week for 20 weeks. At the start of week 20, PDMCs-GFP were intravenously injected at a dose of $2.2 \times 10^{6} / \mathrm{kg}$ body mass of rat in $0.5 \mathrm{ml}$ PS. At the end of 20th week, rats were sacrificed. Group DMH-PS was treated similarly to Group DMH + PDMCs-GFP; however, at the start of week 20,0.5 ml PS was intravenously injected. Group Int + PDMCs-GFP received PS subcutaneously once a week for 20 weeks. At the start of week 20, PDMCs-GFP were intravenously injected at a dose of $2.2 \times 10^{6} / \mathrm{kg}$ body mass of rat in $0.5 \mathrm{ml} \mathrm{PS}$. At the end of 20th week, rats were sacrificed. PDMCs, placenta-derived multipotent cells; DMH, dimethylhydrazine; PS, physiological saline; GFP, green fluorescent protein.

Zeiss AG, Oberkochen, Germany). Paraffin-embedded tissues were deparaffinised in $100 \%$ xylene two times for 5 min each and rehydrated in decreasing concentrations of ethanol: 96,70 and $50 \%$. Slides were boiled in Citrate buffer (10 $\mathrm{mM}$ sodium citrate, $\mathrm{pH}$ 6.0) for $30 \mathrm{~min}$ to retrieve the antigen. Nonspecific reactivity was reduced by incubating tissue sections in a blocking solution (0.1 M PBS with $0.5 \% \mathrm{BSA}$ ) at room temperature for $30 \mathrm{~min}$. Immunostaining was completed following an overnight incubation at $4^{\circ} \mathrm{C}$ with polyclonal goat anti-GFP primary antibody (ab6673; 1:100; Abcam, Cambridge, UK). Following washing three times for $10 \mathrm{~min}$ each with $0.1 \mathrm{M}$ PBS, samples were incubated with donkey anti-goat IgG $(\mathrm{H}+\mathrm{L})$ secondary antibody with Alexa Fluor 488 conjugate (ab150129; 1:1,000; Abcam) for $1 \mathrm{~h}$ at room temperature in the dark. Following nuclear staining with Hoechst 33342 (H3570; 1:5,000; Thermo Fisher Scientific, Inc.), slides were mounted using Mowiol 4-88 (Sigma-Aldrich; Merck KGaA). Confocal analysis was performed using a Zeiss LSM 510 Meta microscope (magnification, x630) and images were captured with Zeiss LSM Image Browser Version 4.2.0.121 software (all from Carl Zeiss AG).

Statistical analysis. The data were analysed using GraphPad Prism 7.0 (GraphPad Software, Inc., La Jolla, CA, USA) using one- or two-way ANOVA followed by Tukey's post-hoc test for multiple comparisons and presented as mean \pm standard deviation. The survival rates were calculated by the Kaplan-Meier method in SPSS 10.0. (SPSS, Inc., Chicago, IL, USA), the

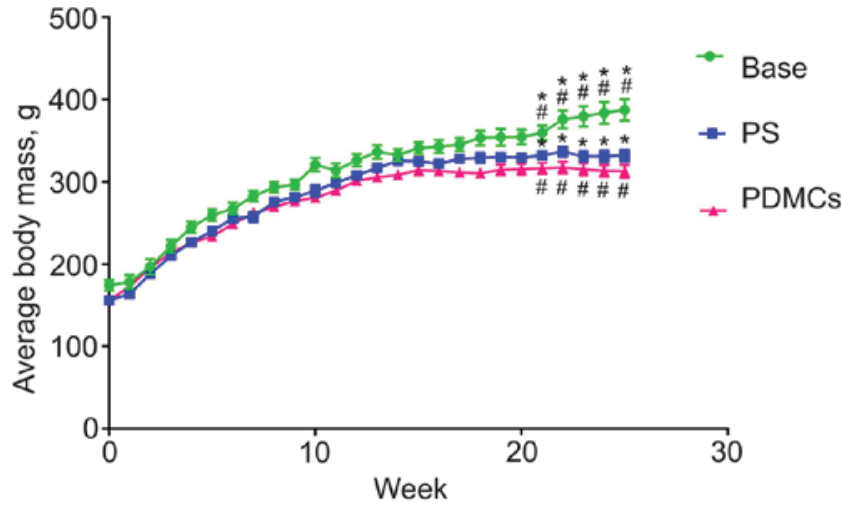

Figure 2. PDMC transplantation does not affect body mass of rats with DMH-induced CC. The results are presented as mean \pm standard deviation and analysed using one-way ANOVA followed by Tukey's post-hoc test. ${ }^{*} \mathrm{P} \leq 0.05$, between base and PS groups; ${ }^{\mathrm{P}} \mathrm{P} \leq 0.05$, between base and PDMCs groups. PDMCs, placenta-derived multipotent cells; DMH, dimethylhydrazine; PS, physiological saline; $\mathrm{CC}$, colorectal cancer.

analysis of survival between two groups was carried out using log-rank test. $\mathrm{P}<0.05$ was considered to indicate a statistically significant difference.

\section{Results}

Effect of transplantation of PDMCs on the body mass, number and area of colon tumours. Body mass of intact 
A

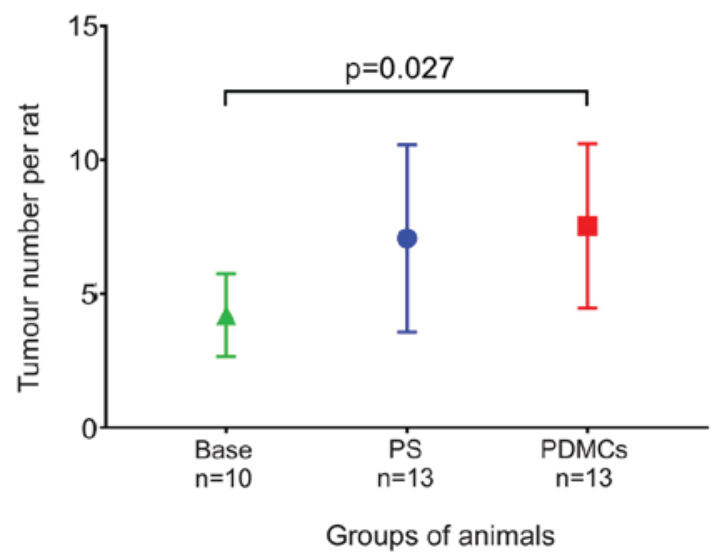

C

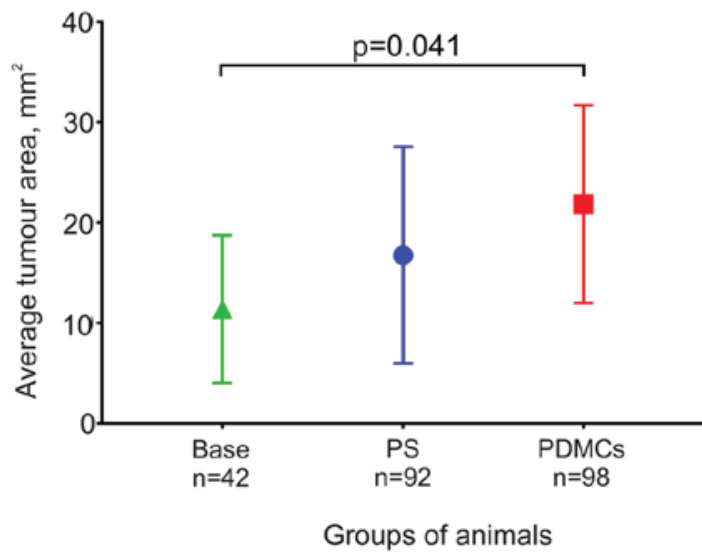

$\mathrm{B}$

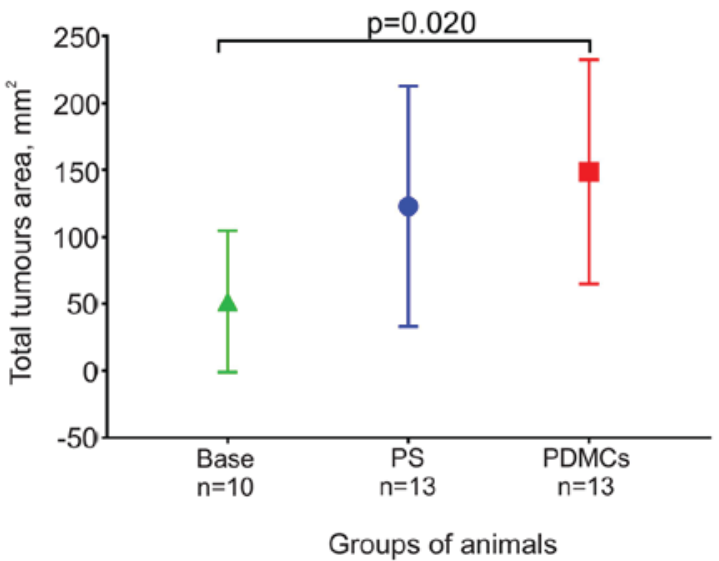

Figure 3. The change in tumour number and tumour dimension parameters in the experimental groups of rats. (A) The number of tumours did not differ significantly in the PDMCs and PS groups. Transplantation of PDMCs causes a slight increase in (B) total area and (C) mean area of the tumours. The results are presented as mean \pm standard deviation. One-way ANOVA followed by Tukey's post-hoc test for multiple comparisons was employed for statistical analysis. PDMCs, placenta-derived multipotent cells; PS, physiological saline.

control rats increased during the 25 weeks of observation, while the masses of animals with DMH-induced cancer began decreasing 16 weeks after the start of $\mathrm{CC}$ induction. At the day of PDMC administration, mean mass of rats in the control and the experimental groups differed significantly (Fig. 2). It is important to note that transplantation of PDMCs did not lead to normalisation of body mass.

The mean number of tumours per rat did not change significantly in animals from the PDMCs and the PS groups, although this number increased significantly during five weeks after PDMCs were transplanted (Fig. 3A).

The total and mean areas of tumours from the PDMCs group were higher than those of the tumours from the PS group, although the difference did not reach statistical significance. The values of these parameters were higher in rats in the PS and PDMCs groups than in the Base group, although a significant increase was noted in animals after PDMC transplantation (Fig. 3B and C).

After DMH-induced development of colon cancer, tumours with different degree of invasion and differentiation were observed, depending on the depth of tumour growth into bowel wall and state of epithelium (Fig. 4A and B). Hence, our next task was to study the effect of intravenous administration of PDMCs on size and number of different colon tumour types (Fig. 4C-F).
The distribution of tumours by the degree of invasion almost did not differ between groups; a significant difference was observed only in rats from the PDMCs and PS groups compared to those in the Base group for the stage T1 tumours (Fig. 4A, invasion into submucosa of the intestine), which is expected, because during 5 weeks the tumours invaded deeper compared to the time of treatment (Fig. 4C).

Measuring the differentiation degree has important prognostic significance and depends on the structure of the colonocytes in the epithelium. Considering the tumour grade distribution by the degree of differentiation of the tumours, a significant difference was observed in moderately differentiated tumours (Fig. 4B) between the PDMCs and Base groups and PS and Base groups (Fig. 4D). Additionally, we observed a tendency towards an increase in the number of less differentiated tumours in the PDMCs and PS groups as compared to those in the Base group (Fig. 4D).

Interestingly, the mean size of the T4 tumours that had invaded the deepest (Fig. 4A) differed between the PDMCs and the PS groups (Fig. 4E), although we did not see a difference in the mean size of tumours with different degrees of differentiation (Fig. 4F).

Distribution of PDMCs in the body. To study the distribution of PDMCs in the rats' bodies, the PDMCs were pre-marked 

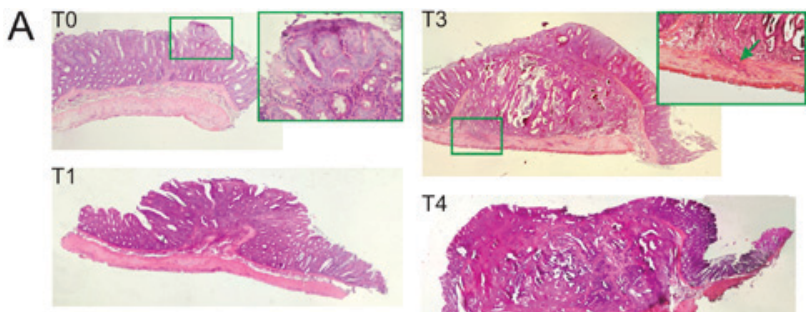

T2
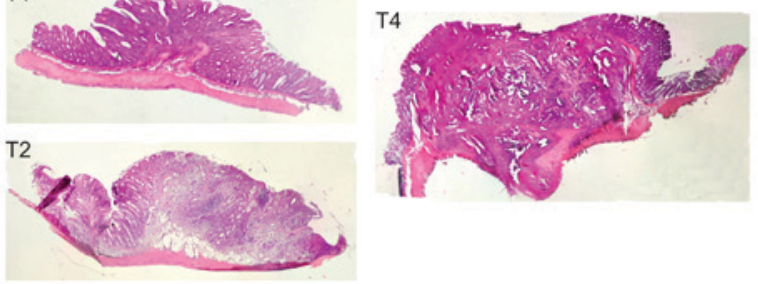

C
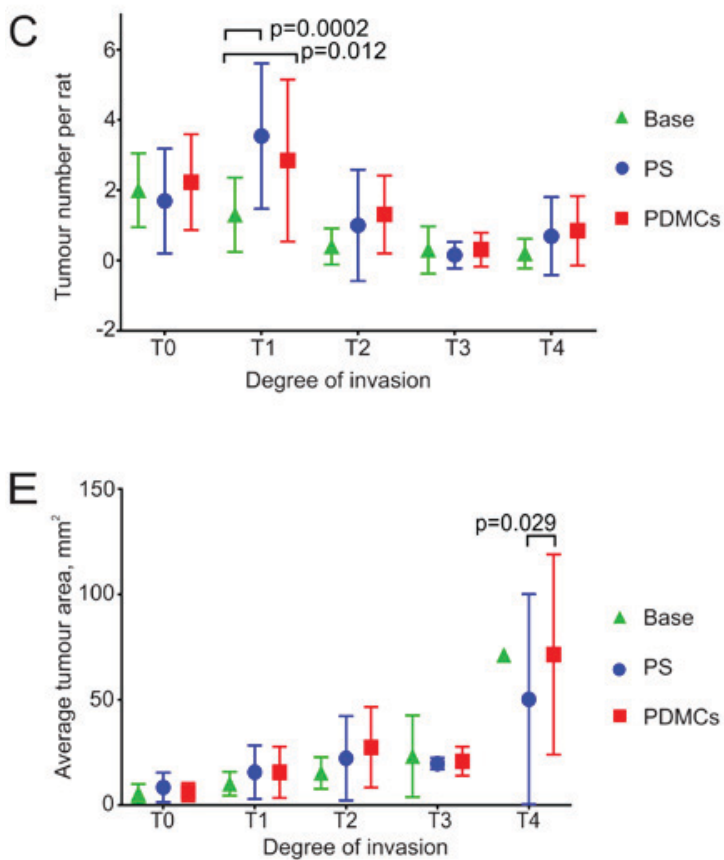
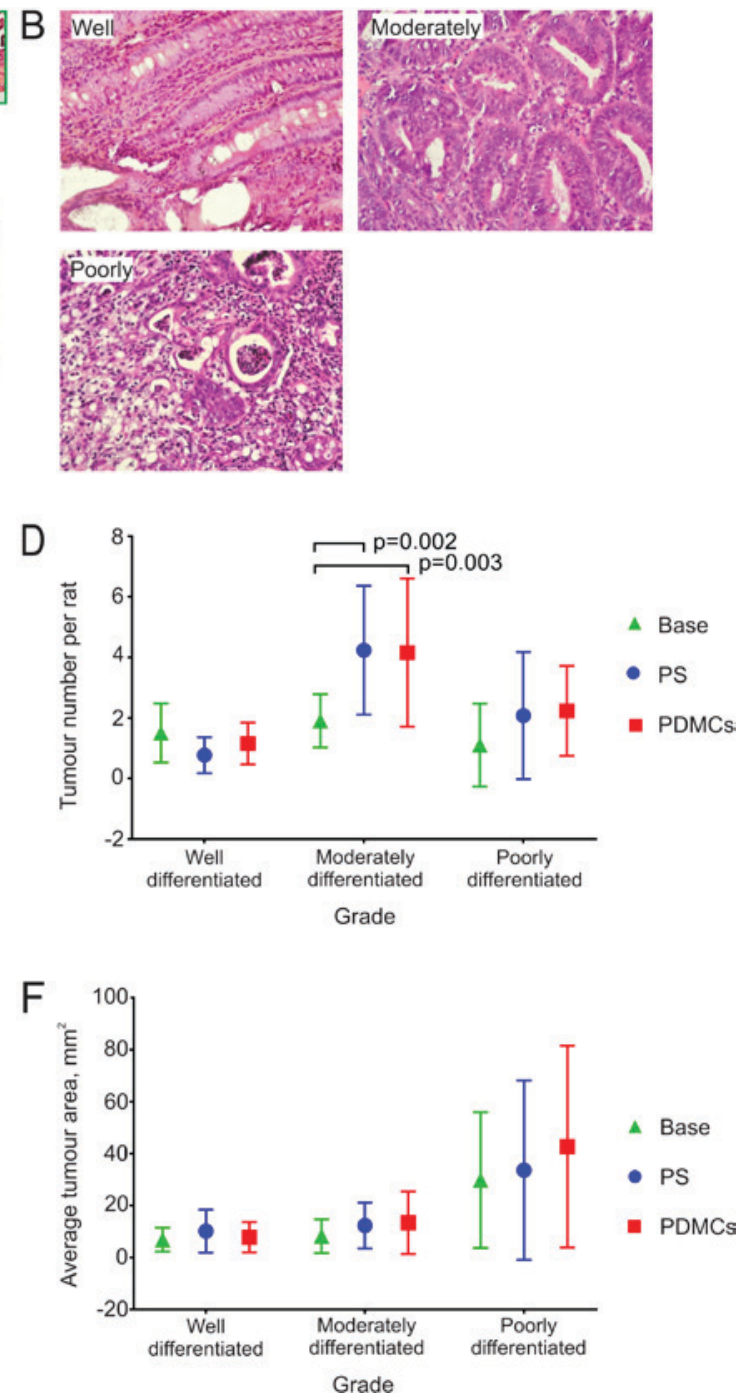

Figure 4. The heterogeneity of colon tumour types and their morphometric parameters in experimental groups. (A) Histological analysis of the DMH induced colon tumours with different degree of invasion into the intestine wall. T0, a tumour is located in the epithelial layer; T1, a tumour reaches tela submucosa; T2, a tumour grows to the muscular layer; T3, a tumour infiltrates tela subserosa (green arrow); T4, a tumour grows into the visceral peritoneum or other organs. H\&E stain, magnification x40, magnification in the green frame T0 x400, magnification in the green frame T3 x200. (B) Grade of tumours was assigned according to epithelium state: well-differentiated tumour (the epithelium contains all types of colon epithelial cells and reflects native colon crypt structure); moderately differentiated (crypt gets deformed, epithelium gets multilayer and/or multicellular instead of unicellular single row); poorly differentiated (there is no native structure and gland forming), H\&E stain, magnification $\mathrm{x} 400$. The number of both tumours at the 1st degree of invasion into the intestinal wall (C) and tumours with moderately differentiated grade (D) increased in PS and PDMCs groups compared to those in Base group. (E) The average area of tumours at the 4th degree of invasion into intestinal wall increased in PDMCs group as compared to PS and Base groups. (F) The average area of tumours at different degrees of differentiation did not change in all experimental groups. Two-way ANOVA followed by Tukey's post-hoc test for multiple comparisons was employed for statistical analysis. The results are presented as mean \pm standard deviation. PDMCs, placenta-derived multipotent cells; DMH, dimethylhydrazine; PS, physiological saline.

by transduction of a genetic vector containing EGFP. The level of transduction of PDMCs was noted to be 7.6 to $57.7 \%$ $(\mathrm{n}=4)$. EGFP was detected in different rat organs on the 7 th day after transplantation (Fig. 5A and Table I). Additionally, alive PDMCs-GFP were visualized by immunohistochemistry in tumour colon tissues (Fig. 5B).

Effect of PDMCs transplantation on the survival rate of rats with $\mathrm{DMH}$-induced $\mathrm{CC}$. One of the most important criteria when estimating the effect of anti-tumour pharmacological and cell preparations is survival analysis. Intravenous allogeneic transplantation of PDMCs led to a reduction in the survival rate of rats with $\mathrm{DMH}$-induced $\mathrm{CC}$ by 17 days (Fig. 6). The median survival rate for the untreated control (PS-S) group was 107 days, and that for the PDMCs-S group was 90 days.
In this study, it was demonstrated that PDMCs administered in rats at the middle phase of colon carcinogenesis, led to an increase in the size of the most invasive tumours and a rise in mortality.

\section{Discussion}

The safety and efficacy of the PDMCs transplantation for the treatment of inflammatory colon diseases have been demonstrated by clinical trials $(13,18)$. Despite the positive results, the effect of PDMCs on the CC, that often accompanies inflammatory processes in the intestine, has not been studied sufficiently. Currently, the two-side effect of MSCs, including placenta-derived MSCs (19), on the growth of transformed cells is a topic of research and some possible mechanisms 
Table I. Measuring EGFP in different rat organs after transplantation of PDMCs-GFP.

\begin{tabular}{lcccccc}
\hline & \multicolumn{2}{c}{ Colon } & & & & \\
\cline { 2 - 4 } Animal group & Tumour & Norm & Liver & Spleen & Lungs & Kidney \\
\hline DMH + PDMCs-GFP & $4 / 5$ & NA & $4 / 5$ & $4 / 5$ & $5 / 5$ & $5 / 5$ \\
DMH + PS & $0 / 5$ & NA & NA & NA & NA & NA \\
Int + PDMCs-GFP & NA & $4 / 4$ & $1 / 4$ & $1 / 4$ & $5 / 5$ & $1 / 4$ \\
\hline
\end{tabular}

Data are presented as the number of rats with a positive signal detected/all rats accepted for analysis. NA, sample absent or not accepted for analysis; PDMCs, placenta-derived multipotent cells; GFP, green fluorescent protein; PS, physiological saline; Int, intact.
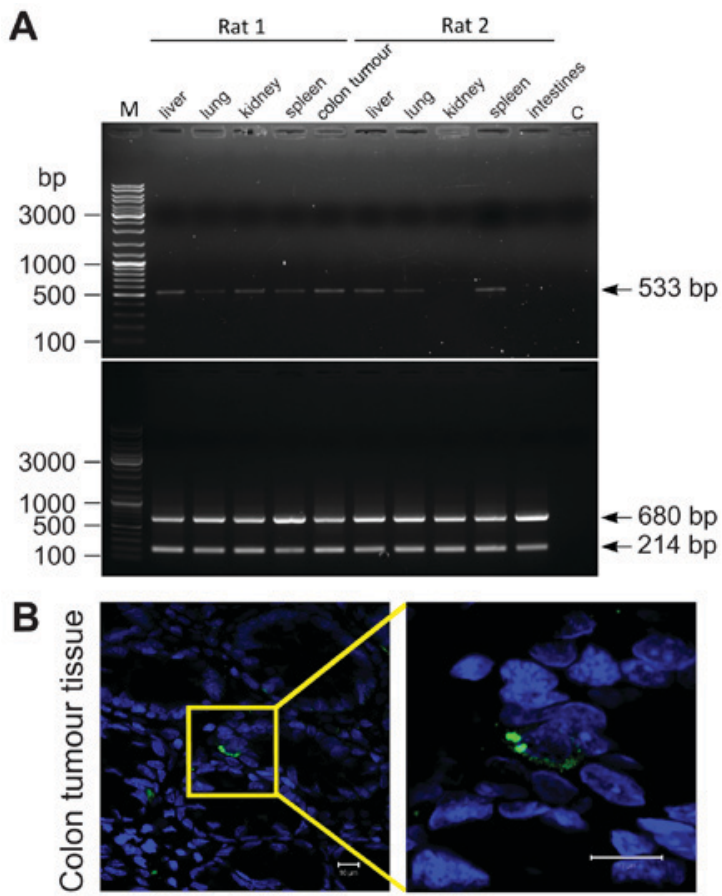

Figure 5. PDMCs engrafted in different rat's organs and colon tumours. (A) A representative example of PDMC-GFP distribution in the rat body one week after administration. A PCR analysis of EGFP (upper panel) and $A C T B$ (bottom panel) in rat's different tissues; rats 1 and 2 refer to groups DMH+PDMCs-GFP and Int+PDMCs-GFP respectively; M, GeneRuler ${ }^{\mathrm{TM}}$ DNA Ladder Mix (Thermo Fisher Scientific, Inc., Waltham, MA, USA), C, water was used as negative control. (B) PDMCs-GFP were identified in rat tumour colon tissues by anti-GFP staining. Immunohistochemistry fluorescence: Anti-GFP are green, nuclei are blue. Scale bar, $10 \mu \mathrm{m}$; magnification, x630. PDMCs, placenta-derived multipotent cells; GFP, green fluorescent protein; EGFP, enhanced green fluorescent protein.

for this phenomenon have been proposed (20,21). Most of the experiments for evaluation of the MSCs' impact on cancer have been carried out in vitro (22) while xenotransplantation models were used in vivo $(23,24)$. In her review, O'Malley et al (25) indicates that most studies regarding the effect of MSCs on CC were performed on immunodeficient animals with allo/xenografted tumours and local administration of high doses MSCs.

The results of such studies cannot be used to predict the impact of MSCs on the development of colon cancer in humans (25). In our study, PDMCs transplanted at middle phase of $\mathrm{CC}$ did not affect either the number or the area of the forming tumours, but detailed analysis of the area

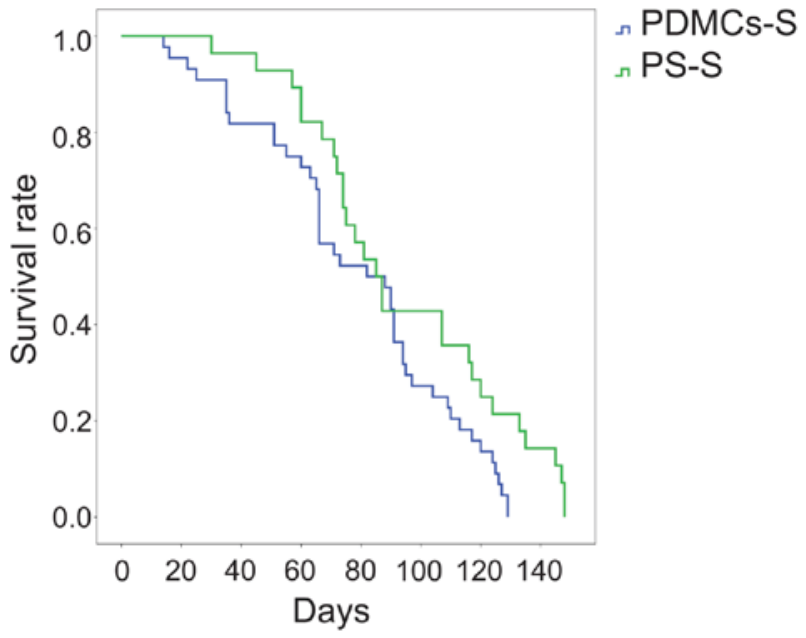

Figure 6. The dynamics of rat survival with DMH-induced $\mathrm{CC}$ after the administration of PDMCs. Log-rank test, $\mathrm{P}=0.015$. PDMCs, placenta-derived multipotent cells; DMH, dimethylhydrazine; $\mathrm{CC}$, colorectal cancer; PS, physiological saline

of tumours depending on the invasion depth revealed an unexpected result due to the selectivity of stimulation of stage T4 tumours. After all, previously, it was shown that after administering bone marrow-derived MSCs at early stages of azoxymethane/dextran sulfate sodium colitis-associated tumourigenesis modelling, the number of tumours decreased, but the mean tumour size did not change $(26,27)$. Katsuno et al observed that after administering bone marrow-derived MSCs to rats in early phase of DMH-induced carcinogenesis, both the number and the volume of generated tumours decreased, while at the late phase it did not have any effect (4).

However, we did not observe any stimulation of colon tumours with low and medium degree of invasion after systemic cell administration that could suggest the selective effect of PDMCs depending on colon cancer cell properties. According to the 'Big Bang' model of CC (28), the colon tumour is heterogenic, that is, it gradually accumulates mutations of different genes. It was recently found that different $\mathrm{CC}$ cell lines are characterized by cell line-specific growth dependency on MSCs; for example, MSCs stimulate the growth of COLO 320, HCT116, and LoVo, and inhibit the growth of DLD-1, HCT-15, and HT-29 if they are xenotransplanted to SCID mice (29). Bearing in mind that the aforementioned cell lines differ in mutation and gene 
expression profiles, one can propose that spontaneous colon tumours at stage T4 contain cells that would be able to answer the contact-dependent and/or paracrine effects of PDMCs. A similar contrasting effect was shown after co-transplantation of Wharton's jelly MSC (WJMSCs) with cancer stem cell (CSCs) lines from different types of lung tumours (30). The authors suggested that the stimulatory or inhibitory effect of MSCs on pulmonary tumours depends on the degree of its differentiation. It was also found that the co-transplantation of WJMSCs with the LCSC lines in the NOD/SCID mice led to the elevation in the expression of the CSCs marker CD133 indicating a reduction in the degree of differentiation of the tumours, and so an increase of their growth and metastasis (30).

We showed that PDMCs were able to engraft into tissues of various organs-the liver, spleen, lungs, kidneys, colon, and colon tumours. The engraftment of human PDMCs after intravenous transplantation was detected earlier in different organs (31) and colon cancer (14) of rodents. Also using the immunohistochemistry method to detect GFP-labelled PDMCs, we showed the presence of the alive PDMCs in the tissue of spontaneous colon tumour on day 35 after administration (14). Since the traces of PDMCs were detected in many different organs, we must take into account not only the interaction between MSCs and various cell populations of the tumour (endotheliocytes, epitheliocytes, tumour-associated macrophages, and cancer-associated fibroblasts), but also the complex effect of MSCs on the organism as a whole that changes the functioning of other organ systems, which can reflect on carcinogenesis.

Particularly, along with stimulation of the tumour growth at stage $\mathrm{T} 4$, we found deterioration in animal survival rate after PDMC transplantation that has not been established until now. Taking into account the fact that, as mentioned earlier, the total number and area of tumours does not change at the same time, it indicates the necessity to study MSCs effect on different spontaneous colon tumours which are as usual very heterogenic. Furthermore, the animal survival rate as a critical indicator has to be evaluated before postulating a conclusion about the effect of MSC on carcinogenesis. It should be noted that the effect of MSC transplantation on animal survival with carcinogenesis of the colon has almost not been investigated. Only Shinagawa et al in their study showed that co-transplantation of colon cancer cell line KM12SM and MSCs led to a decrease of mice survival rate as compared to animals with KM12SM alone (5). Decreased survival of mice after co-transplantation may be caused by the enhancement of proliferation, angiogenesis, migration, and invasion along with the inhibition of apoptosis of tumour cells by MSCs (5).

Further investigations designed to explore the mechanisms underlying the effects of PDMCs on different colon tumour subtypes may shed light on the complex effects of MSC systemic transplantation on cancer progression.

\section{Acknowledgements}

The authors thank Mr. S. Martynenko and Dr. M. Sokolov for their help in organisation and support of this research. This study was supported by the Institute of Cell Therapy and The National Academy of Sciences of Ukraine (grant no. 0114U003877).

\section{References}

1. Squillaro T, Peluso G and Galderisi U: Clinical trials with mesenchymal stem cells: An update. Cell Transplant 25: 829-848, 2016.

2. Mele V, Muraro MG, Calabrese D, Pfaff D, Amatruda N, Amicarella F, Kvinlaug B, Bocelli-Tyndall C, Martin I, Resink TJ, et al: Mesenchymal stromal cells induce epithelial-to-mesenchymal transition in human colorectal cancer cells through the expression of surface-bound TGF- $\beta$. Int J Cancer 134: 2583-2594, 2014.

3. De Boeck A, Pauwels P, Hensen K, Rummens JL, Westbroek W, Hendrix A, Maynard D, Denys H, Lambein K, Braems G, et al: Bone marrow-derived mesenchymal stem cells promote colorectal cancer progression through paracrine neuregulin 1/HER3 signalling. Gut 62: 550-560, 2013.

4. Katsuno T, Ochi M, Tominaga K, Tanaka F, Sogawa M, Tanigawa T, Yamagami H, Shiba M, Watanabe K, Watanabe T, et al: Mesenchymal stem cells administered in the early phase of tumorigenesis inhibit colorectal tumor development in rats. J Clin Biochen Nutr 53: 170-175, 2013.

5. Shinagawa K, Kitadai Y, Tanaka M, Sumida T, Kodama M, Higashi Y, Tanaka S, Yasui W and Chayama K: Mesenchymal stem cells enhance growth and metastasis of colon cancer. Int $\mathrm{J}$ Cancer 127: 2323-2333, 2010.

6. Altobelli E, D'Aloisio F and Angeletti PM: Colorectal cancer screening in countries of european council outside of the EU-28. World J Gastroenterol 22: 4946-4957, 2016.

7. American Cancer Society: Colorectal Cancer Facts \& Figures 2014-2016. https://ww w.cancer.org/content/dam/cancer-org/ research/cancer-facts-and-statistics/colorectal-cancer-facts-andfigures/colorectal-cancer-facts-and-figures-2014-2016.pdf.

8. Jackstadt R and Sansom OJ: Mouse models of intestinal cancer. J Pathol 238: 141-151, 2016.

9. Perše M and Cerar A: Morphological and molecular alterations in 1,2 dimethylhydrazine and azoxymethane induced colon carcinogenesis in rats. J Biomed Biotechnol 2011: 473964, 2011.

10. Dominici M, Le Blanc K, Mueller I, Slaper-Cortenbach I, Marini F, Krause D, Deans R, Keating A, Prockop Dj and Horwitz E: Minimal criteria for defining multipotent mesenchymal stromal cells. The international society for cellular therapy position statement. Cytotherapy 8: 315-317, 2006.

11. Mariotti E, Mirabelli P, Abate G, Schiattarella M, Martinelli P, Fortunato G, Di Noto R and Del Vecchio L: Comparative characteristics of mesenchymal stem cells from human bone marrow and placenta: CD10, CD49d and CD56 make a difference. Stem Cells Dev 17: 1039-1041, 2008.

12. Miao Z, Jin J, Chen L, Zhu J, Huang W, Zhao J, Qian H and Zhang X: Isolation of mesenchymal stem cells from human placenta: Comparison with human bone marrow mesenchymal stem cells. Cell Biol Int 30: 681-687, 2006.

13. Hu J, Zhao G, Zhang L, Qiao C, Di A, Gao H and Xu H: Safety and therapeutic effect of mesenchymal stem cell infusion on moderate to severe ulcerative colitis. Exp Ther Med 12: 2983-2989, 2016.

14. Svitina H, Kyryk V, Skrypkina I, Kuchma M, Bukreieva T, Areshkov P, Shablii Y, Denis Y, Klymenko P, Garmanchuk L, et al: Placenta-derived multipotent cells have no effect on the size and number of DMH-induced colon tumors in rats. Exp Ther Med 14: 2135-2147, 2017.

15. Kean TJ, Lin P, Caplan AI and Dennis JE: MSCs: Delivery routes and engraftment, cell-targeting strategies and immune modulation. Stem Cells Int 2013: 732742, 2013.

16. Kurtz A: Mesenchymal stem cell delivery routes and fate. Int J Stem Cells 1: 1-7, 2008.

17. Zufferey R, Nagy D, Mandel RJ, Naldini L and Trono D: Multiply attenuated lentiviral vector achieves efficient gene delivery in vivo. Nat Biotechnol 15: 871-875, 1997.

18. Zhang J, Lv S, Liu X, Song B and Shi L: Umbilical cord mesenchymal stem cell treatment for crohn's disease: A randomized controlled clinical trial. Gut Liver 12: 73-78, 2018.

19. Silini AR, Cancelli S, Signoroni PB, Cargnoni A, Magatti M and Parolini O: The dichotomy of placenta-derived cells in cancer growth. Placenta 59: 154-162, 2017.

20. Rhee KJ, Lee JI and Eom YW: Mesenchymal stem cell-mediated effects of tumor support or suppression. Int J Mol Sci 16: 30015-30033, 2015.

21. Klopp AH, Gupta A, Spaeth E, Andreeff M and Marini F III: Concise review: Dissecting a discrepancy in the literature: Do mesenchymal stem cells support or suppress tumor growth? Stem Cells 29: 11-19, 2011. 
22. Chen D, Liu S, Ma H, Liang X, Ma H, Yan X, Yang B, Wei J and Liu X: Paracrine factors from adipose-mesenchymal stem cells enhance metastatic capacity through Wnt signaling pathway in a colon cancer cell co-culture model. Cancer Cell Int 15: 42, 2015.

23. Ohta N, Ishiguro S, Kawabata A, Uppalapati D, Pyle M, Troyer D, De S, Zhang Y, Becker KG and Tamura M: Human umbilical cord matrix mesenchymal stem cells suppress the growth of breast cancer by expression of tumor suppressor genes. PLoS One 10: e0123756, 2015.

24. Zheng H, Zou W, Shen J, Xu L, Wang S, Fu YX and Fan W: Opposite effects of coinjection and distant injection of mesenchymal stem cells on breast tumor cell growth. Stem Cells Transl Med 4: 1216-1228, 2016.

25. O'Malley G, Heijltjes M, Houston AM, Rani S, Ritter T, Egan LJ and Ryan AE: Mesenchymal stromal cells (MSCs) and colorectal cancer: A troublesome twosome for the anti-tumour immune response? Oncotarget 7: 60752-60774, 2016

26. Nasuno M, Arimura Y, Nagaishi K, Isshiki H, Onodera K, Nakagaki S, Watanabe S, Idogawa M, Yamashita K, Naishiro Y, et al: Mesenchymal stem cells cancel azoxymethane-induced tumor initiation. Stem Cells 32: 913-925, 2014.

27. Chen Z, He X, He X, Chen X, Lin X, Zou Y, Wu X and Lan P. Bone marrow mesenchymal stem cells ameliorate colitis-Associated tumorigenesis in mice. Biochem Biophys Res Commun 450: 1402-1408, 2014.
28. Sottoriva A, Kang H, Ma Z, Graham TA, Salomon MP, Zhao J, Marjoram P, Siegmund K, Press MF, Shibata D and Curtis C: A Big Bang model of human colorectal tumor growth HHS Public Access new model provides a quantitative framework to interpret tumor growth dynamics and the origins of ITH with significant clinical implications. Nat Genet 47: 209-216, 2015.

29. Nakagaki S, Arimura Y, Nagaishi K, Isshiki H, Nasuno M, Watanabe S, Idogawa M, Yamashita K, Naishiro Y, Adachi Y, et al: Contextual niche signals towards colorectal tumor progression by mesenchymal stem cell in the mouse xenograft model. J Gastroenterol 50: 962-274, 2015.

30. Vulcano F, Milazzo L, Ciccarelli C, Eramo A, Sette G, Mauro A, Macioce G, Martinelli A, La Torre R, Casalbore P, et al: Wharton's jelly mesenchymal stromal cells have contrasting effects on proliferation and phenotype of cancer stem cells from different subtypes of lung cancer. Exp Cell Res 345: 190-198, 2016.

31. Wu CG, Zhang JC, Xie CQ, Parolini O, Silini A, Huang YZ, Lian B, Zhang M, Huang YC and Deng L: In vivo tracking of human placenta derived mesenchymal stem cells in nude mice via ${ }^{14} \mathrm{C}$-TdR labeling. BMC Biotechnol 15: 55, 2015.

This work is licensed under a Creative Commons Attribution-NonCommercial-NoDerivatives 4.0 International (CC BY-NC-ND 4.0) License. 